\title{
A Contribution of Tipler's Omega Point Theory to Near-Death Studies
}

\author{
James C. Crumbaugh, Ph.D. \\ Biloxi, $M S$
}

ABSTRACT: A fundamental principle of behavioral and natural scientists is reductionism: all mental phenomena can be reduced to a physical basis. Phenomena that have no physical basis cannot really exist. For most scientists this rules out transpersonal, spiritual or noetic, and religious phenomena, all of which maintain strongly antireductionist positions. Thus near-death researchers have an uphill battle to stay scientifically afloat. However, mathematician Frank Tipler argues that, while reductionism is necessary to the scientific world, it does not negate the religious, noetic, or spiritual dimension of human experience. He demonstrates by hard-core physics the existence of God and religious and spiritual phenomena. While the proofs he offers can be understood only by other astrophysicists, his overall viewpoint is comprehensible by laypeople. I present his concepts and arguments, and highlight the value of this orientation for near-death studies. Tipler's work takes the steam out of scientific rejection of religious, spiritual, or noetic phenomena, and makes it possible to accept these phenomena while maintaining a strictly scientific posture. Near-death researchers can gain a greater degree of scientific acceptance by adopting Tipler's position on reductionism.

The Omega Point Theory defines The Physics of Immortality (Tipler, 1994), in which Frank Tipler, Professor of Mathematics at Tulane University, used hard-core physics and mathematics as a solution to prove the probability of survival of personality after death. The course of events predicted by this theory is consistent with the most common basic events reported in the literature on near-death experiences (NDEs).

James C. Crumbaugh, $\mathrm{Ph} . \mathrm{D}$., is a clinical psychologist in private practice since VA retirement. This paper is based on a paper presented at the International Association for Near-Death Studies Annual Conference in San Antonio, TX, in November, 1997. Reprint requests should be addressed to Dr. Crumbaugh at 140 Balmoral Avenue, Biloxi, MS 39531-4701. 
Throughout the ages the most difficult part of the survival hypothesis has been the development of a physics that would permit personality to survive death of the physical body. The majority of philosophers as well as behavioral and physical scientists have rejected the possibility of survival on the grounds of this failure. Most of the remainder have simply assumed, as many believing religionists do, that such a physics exists but is beyond human understanding and therefore not to be questioned.

A few scientists have continued to struggle with the problem. Among them have been Kenneth Ring (1982), who proposed the original idea that personality is carried by patterns of interacting wave phenomena much like those used in producing a hologram, and that the entire coding pattern can be reproduced by any small portion of that interference pattern that can be obtained. The late C. J. Ducasse, Professor of Philosophy at Brown University, employed the concept of a "psychic double," but did not theorize specifically as to its nature (Crumbaugh, 1956).

Raymond Wheeler (1935) and Gardner Murphy (1945) used an analogy stemming from the gestalt psychologists' concept of transposition, in which interacting energy fields follow a pattern isomorphic to the personality pattern, as a map is isomorphic to a country but not of the same material nature. Of course, the nature of these interacting energy fields remains unknown, though it is presumably a type of interaction between wave motions in energy fields that act in a similar fashion to those in the brain. I have extended this analogy to what happens in a tape recording (Crumbaugh, 1956). Let us say that Judy Garland's singing "Over the Rainbow" is recorded on a tape, and the tape is destroyed. One can recreate her voice at any time by a process analogous to but not identical with her vocal cords. This process may occur in a phonograph record, a motion picture film, or a videotape, and so forth. They all can reproduce the music pattern and Garland's vocal timbre to the extent that, by listening alone, one could not determine whether it were a recording or really she.

Now if the record is destroyed, the potential to recreate the voice and song exists as long as there is another recording in some form. But what if no such form can be found? Is this the end of Garland? Not if the number of songs and voices is finite, so that any combination could be reproduced by reproducing all possible combinations. And the number is finite, both as to music and as to personalities.

But one may ask-or perhaps gasp-whether I am suggesting that one could resurrect Garland by recreating not only everyone who ever 
did live, but also everyone who ever could have lived? Yes, I am suggesting something like that, though one could before completing the reproductions cull out many of the unwanted ones by restricting the known parameters. For example, one might limit reproduction to the pitch range of the song, or the time signature, and so forth. Still a fantastic number of songs would be left, many of which had never been composed previously by human beings. Would all of these be recreated? Yes, they would; though the unwanted ones could then be destroyed. And, of course, in this total process a higher power must exist to carry out the resurrections and then the selections. And in this analogy we are the higher power.

But, one may shout, are we going to murder all of these never-beforeexistent human beings that we created? No; we could, but we wouldn't, unless these individuals wanted to be exterminated. But who would feed, clothe and house all of these resurrected beings, even all of those who did exist? Tipler's answer is that the Omega Point would. And it would take our place as the higher power, or God. Manipulations of mass and energy-which are potentially interchangeable as in the formula $\mathrm{E}=\mathrm{mc}^{2}$-could utilize all of the cosmic materials, including stars, comets, planets, galaxies, and so forth, that were not already used in the resurrection process. The whole Omega Point process is a supercosmic utilization of everything that exists. The concepts of Ring, Wheeler, Murphy, Crumbaugh, and Ducasse can all be seen as analogous to the physical concepts of Omega Point Theory.

So what is this mysterious Omega Point? Does it have any physical reality, or is it just a mathematical concept? It is both. In The Physics of Immortality, Tipler (1994) delineated the future of the human race, indeed of all life in the universe and of the entire cosmos, showing that the physics of it all is consistent throughout cosmic history, and that the Big Bang of some 15 billion years ago will culminate some 85 billion years in the future in a colossal crash. This crash represents what the Jesuit philosopher Pierre Teilhard de Chardin has described as the end point, or Omega Point, the Big Bang being the beginning or Alpha Point. Of course, time estimates may differ wildly, but at present the "closed universe" cosmologists seem to favor a span of some 100 billion years between the Alpha and the Omega Points. And Tipler's theory will work only if it is a closed universe-that is, if the universe reaches a critical point of expansion and then "crashes." If it is an eternally expanding universe or an "open" universe, there is no hope for life in the long run: everything will eventually grow cold and die out. But in spite of some recent studies favoring an open universe, 
at present the majority of astrophysicists seem to favor the closed universe concept. If true, the Omega Point can change human history, resurrect the dead, and create the second Eden, this time one of eternal bliss.

How does this relate to near-death studies? It relates through the fact that if Omega Point Theory is correct, the most commonly described NDE phenomena can be delineated under it. To understand this, let us sketch the basic principles of this theory and examine the elements that are common to both it and the typical NDE. The first and biggest advantage of Tipler's argument is that it accepts the noetic or spiritual dimension of human experience on the one hand, while on the other hand embracing a fundamental principle of both behavioral and physical hard sciences: reductionism, which most scientists consider essential to scientific understanding.

The concept of reductionism goes back to British Empiricist philosopher John Locke's dictum that "Nothing is in the intellect which is not first in the senses." In other words, all mental processes that really exist have a physical basis, and if religious concepts like the "soul" cannot be detected by physical means in a physical medium, they cannot actually exist. Religious, spiritual, or noetic points of view usually have hit a scientific stone wall by maintaining an antireductionist position.

Thus a wedge has long been driven between hard science and religious/spiritual phenomenological concepts, with an uneasy peace that has boiled down to "live and let live": religion is allowed on Sunday, provided we return to science on Monday. But Tipler argues that both reductionist and spiritual concepts are valid. He does this by showing that while all that is real is physical, the "spirit" is also physical. No, there is not any "soul" floating around without any material medium: in fact, the spirit ceases to exist when the body dies. But at the Omega Point it will be resurrected, even though the entire process is physical. It might be noted here that the late Herbert Armstrong, founder of the World Wide Church of God, had a similar concept.

So how do we get to this Omega Point? First, we must get used to the fact that, as both philosophers and scientists, as well as most theologians of both mainstream Christianity and Judaism have agreed, experienced time is a human construct that does not exist objectively. Of course, chronos or "proper" or siderial or "star" time functions apart from human experience. But what the Greeks called kairos or experienced time, in which a "siderial hour" may seem forever in a dentist's chair but only a moment in the arms of a loved one, ceases to exist at the 
moment of death. Therefore the approximately 15 billion years that the cosmologists generally seem to estimate since the Big Bang creation of the universe may be only a short blip on a radar screen in comparison with the 85 or so billion years that lie ahead before we reach the Omega Point, the "end of time" or collapse and crash of the universe, the point at which Tipler said the resurrection will occur.

What creates this collapse? As noted on page 7, a preliminary axiom is that the universe is "closed," rather than "open" or expanding forever. The collapse follows from the universe's expanding to the point at which its critical mass is balanced by the gravitational strength of withdrawal from the expansion. Upon reaching this Omega Point, there is a "crash" due to a sort of "zero point" at which Tipler predicts, from known laws of physics, that certain fantastic things will happen. To understand this we must back up a bit to see what has been going on during the progress toward this Omega Point.

Tipler holds that our future belongs to a New World of cosmic travel unveiled by the astronauts, just as our present belongs to the New World opened up by Christopher Columbus. We will be forced by cosmic activity to abandon Earth and use its material elsewhere. During the 85 or so billion years before the "end of time" in the siderial sense, fantastic developments of the quantum computer now on the drawing board will make possible the resurrection of all deceased personalities by simulation, the approximate regeneration of all bodies previously alive, followed by emulation, the regeneration in exact detail, at which point conscious experience will resume under the "Rip Van Winkle effect," as if no time has passed since death.

But what if the human race destroys itself by the atomic bomb before ever getting close to this end point? Tipler held that what is known as the "Eternal Life Postulate" will prevent this from happening. This postulate, like the Omega Point Theory itself, is far too complex to attempt to explain here, for only advanced mathematical physicists and cosmologists could follow Tipler's development of the advanced formulas upon which these concepts rest. Tipler presents the data by which such advanced scientists can follow and verify the logic of his conclusions; he occupies the last third of his book with these. The general meanings behind the many equations are comparatively easy to grasp, however, and Tipler wrote that anyone with a high school education should be able to follow them.

Whether or not the latter is true, the general reader will find his book fascinating and will marvel at the depth of scholarship through which Tipler encompassed almost every type of cosmic and astrophysical 
concept, as well as virtually all systems of religion and philosophy in relation to each of the concepts in his theory. At this point the reader may be again asking just how such fantastic cosmology can relate to NDEs. The answer is that the passage of siderial or cosmological time from present death to the Omega Point involves the extensiveness of "black holes," dark matter that is too dense and therefore too heavy for light to escape from it, and cosmic travel through such space and eons of siderial time; and that everything that happens during those eons is compressed by the Rip Van Winkle effect to the kairos or experienced time of no more than going through a dark tunnel to the bright light and brilliant expanse of a New Life. This is predicted both by Omega Point Theory and noetic science as the end result, in which formerly living personalities are recreated.

Is this not a parallel to the most commonly reported type of NDE? And these formerly living personalities are not only recreated, but also, through the emulation process, recreated as the individual he or she wishes to be: one can choose to have a perfect body with all defects corrected, and as sexually attractive as one desires. Sex exists in Heaven, but there is no conflict over it, as each person can choose to have a perfect partner. The Omega Point will compute and produce this for each individual. And there is a "purgatory" through which those who have lived "badly," in ways that are inconsistent with social welfare, are given a chance to travel. They will be required to choose either "purification" or eternal destruction (permanent death).

How all of this can work from the standpoint of hard-core physics is beyond the scope of the present paper. One must read Tipler's book to appreciate that he is not only serious but competent in presenting his work and in demonstrating the physics behind it, as well as presenting possible experimental designs that would test it.

I was granted an interview with Tipler, and as a result can testify that he is a level-headed person who not only believes passionately in his work but also can support it well through fingertip knowledge of every facet of the physics involved. Since I did not connect Omega Point Theory with NDEs at the time of that interview, I did not elicit Tipler's views on these phenomena. But it appears that NDE concepts can fit this theory without damage to either, and near-death researchers can gain an advantage through acceptance of reductionism without loss of the noetic or spiritual dimension. The latter can bring near death studies into the scientific community and thereby gain a higher level of scientific respectability, which will create a more receptive attitude on the part of scientific journals for publication of near-death studies. 
It seems clear furthermore that the study of Omega Point Theory may be a possible source of new leads in the development of NDE theory.

\section{References}

Crumbaugh, J. C. (1956). The significance of gestalt psychology for the problem of immortality. Journal of the American Society for Psychical Research, 50, 59-65.

Murphy, G. (1945). Field theory and survival. Journal of the American Society for Psychi. cal Research, 39, 181-209.

Ring, K (1980). Life at death: A scientific investigation of the near-death experience. New York, NY: Coward. McCann and Geoghegan.

Tipler, F. J. (1994). The physics of immortality. New York, NY: Anchor/Doubleday.

Wheeler, R. (1935). Organismic versus mechanistic logic. Psychological Review, 42, 335352. 